\title{
NÍVEL DE POTÊNCIA SONORA NAS OPERAÇÕES AGRÍCOLAS
}

\author{
ARCOVERDE, Salvio Napoleão Soares ${ }^{1}$ \\ CORTEZ, Jorge Wilson ${ }^{2}$ \\ PITANGA JÚNIOR, Clóvis de Oliveira ${ }^{3}$ \\ NAGAHAMA, Hideo de Jesus ${ }^{4}$
}

\begin{abstract}
RESUMO: O ruído gerado pela mecanização agrícola trouxe efeitos colaterais, e um deles é o dano à audição do operador de máquinas agrícolas. A NR-15 estabelece os limites de tolerância para os ruídos contínuos, sendo a exposição máxima permitida ao nível de ruído de $115 \mathrm{~dB}$ (A) para indivíduos que não estejam utilizando equipamentos de proteção individuais e $85 \mathrm{~dB}$ (A), considerando uma jornada de trabalho de $8 \mathrm{~h}$. O objetivo deste trabalho foi estudar a influência da velocidade de trabalho e a condição do solo nas operações agrícolas na determinação dos níveis de potência sonora emitido pelo trator. O trabalho foi realizado no Centro de Ciências Agrárias da UNIVASF, Petrolina/PE utilizando um trator Valtra modelo 785 TDA sem cabine e o medidor do nível de potência sonora. Avaliou-se o nível de potência sonora do trator em cinco velocidades de deslocamento $\left(3,0 ; 5,0 ; 8,7 ; 10,7 \mathrm{e} 13,0 \mathrm{~km} \mathrm{~h}^{-1}\right)$ sob solo preparado e solo coberto com plantas daninhas, com os equipamentos: grade leve em tandem, grade leve off-set com discos de 55,8 cm, grade leve off-set com discos de $60,8 \mathrm{~cm}$, escarificador, roçadora e distribuidor pendular. Os valores mais relevantes foram encontrados no trator e na operação de gradagem, observando-se que a exigência de potência no motor aumentou o nível de potência sonora.
\end{abstract}

Palavras-chave: Máquinas agrícolas. Decibelímetro. Ruído.

\section{SOUND POWER LEVEL IN AGRICULTURAL OPERATIONS}

\begin{abstract}
SUMMARY: The noise generated by agricultural mechanization brought side effects, and one of them is the damage the hearing of the operator of agricultural machinery. The NR-15 establishes the limits of tolerance for continuous noise, and exposure to the maximum allowable noise level of $115 \mathrm{~dB}$ (A) for individuals who are not using individual protection equipment and $85 \mathrm{~dB}(\mathrm{~A})$, considering a shift work of $8 \mathrm{~h}$. The objective was to study the influence of working speed and soil condition in agricultural operations in the determination of sound power levels emitted by the tractor. The study was conducted at the Center for Agricultural Sciences - UNIVASF, Petrolina / PE using a Valtra tractor model 785 TDA cabin and meter without the sound power level. We evaluate the sound power level of the tractor in five speeds $\left(3.0,5.0,8.7,10.7\right.$ and $\left.13.0 \mathrm{~km} \mathrm{~h}^{-1}\right)$ on prepared soil and ground covered with weeds, with the equipment: lightweight tandem grid, grid light off-set with discs of $55.8 \mathrm{~cm}$, grade mild off-set with discs of $60.8 \mathrm{~cm}$, chisel plow, mower and distributor commuting. The most significant amounts were found in the tractor and plow in operation, noting that the requirement of increased power in the engine sound power level.
\end{abstract}

Key words: Agricultural machinery. Decibel. Noise.

\footnotetext{
${ }^{1}$ Aluno de graduação em Engenharia Agrícola e Ambiental na Universidade Federal do Vale do São Francisco UNIVASF - Juazeiro, BA. salviounivasf@ hotmail.com

${ }^{2}$ Eng. Agrônomo, Prof. Adjunto, Colegiado de Engenharia Agronômica, Universidade Federal do Vale do São

Francisco - UNIVASF, Petrolina, PE. jorge.cortez@univasf.edu.br

${ }^{3}$ Aluno de graduação em Engenharia Agrícola e Ambiental na Universidade Federal do Vale do São Francisco UNIVASF - Juazeiro, BA. bangato@gmail.com

${ }^{4}$ Eng. Agrônomo, Chefe da Seção de Apoio as Atividades de Campo da Universidade Federal do Vale do São

Francisco - UNIVASF, Petrolina, PE. hideo.nagahama@ univasf.edu.br
} 


\section{INTRODUÇÃO}

Os altos níveis sonoros e a elevada potência e dimensões das máquinas agrícolas, visando maior desempenho nas operações, são causas que aguçam o aumento das doenças auditivas dos operadores no trabalho (MARTINELI, 2008).

Fernandes (2003) aponta que outras causas são destacadas na condição de insalubridade dos operadores de tratores como a vibração, insolação, poeira, defensivos agrícolas, insetos e calor do motor. O autor ainda destaca no presente estudo que $60 \%$ dos operadores apresentaram perda auditiva.

Simone et al. (2006) citado por Cunha, Duarte e Rodrigues (2009) avaliaram as principais fontes de potência sonora em máquinas agrícolas, sendo o escape, que causa grande intensidade, responsável por 45 a $60 \%$ do ruído total. Além disso, a aspiração, ventilador e vibração, respectivamente, ficaram com o percentual de (15-20\%), (12-20\%), (15-20\%) do total da potência sonora.

Fernandes (1993) em estudo comparativo sobre níveis de potência sonora em tratores de marcas e modelos diferentes, e com potências muito próximas, operando quatro implementos, evidenciou que em $91 \%$ das medições a potência sonora foi maior nas marchas mais longas; em $84 \%$ a potência sonora foi proporcional a rotação do motor; em $94 \%$ dos ensaios a potência sonora foi maior em solo seco. Quanto ao implemento, a operação com subsolador apresentou maior média de potência sonora 99,26 dB(A), acompanhada pela aração 97,64 dB (A), escarificação 97,36 dB (A), e gradagem 96,65 dB (A).

Delmond e Reis (2006) analisaram o nível de potência sonora em um trator agrícola em operação de arado e, concluíram que o aumento da velocidade de $5,1 \mathrm{~km} \mathrm{~h}^{-1}$ e rotação de $1.800 \mathrm{rpm}$ (rotações por minuto) aumentam significativamente o nível de potência sonora. Ainda os autores apontam que a operação de gradagem foi realizada acima do limite máximo de $85 \mathrm{~dB}(\mathrm{~A})$, e que não foi observado o uso de equipamentos de proteção auriculares.

Estudos sobre níveis de ruído em operações agrícolas contribuem para investigar a real necessidade de se utilizar medidas de conforto e segurança para o operador, como a utilização de equipamentos de proteção individual, conhecidos como protetores auriculares, para coibir os ruídos. Entretanto, para isto, deve-se observar as condições ambientais locais somados a influência de cada operação agrícola realizada, no comportamento dos ruídos.

Diante do exposto, tomou-se como objetivo geral para este trabalho avaliar o nível de potência sonora emitido por trator em operações agrícolas em função da velocidade e da condição do solo. 


\section{MATERIAL E MÉTODOS}

O trabalho foi conduzido no Centro de Ciências Agrárias da UNIVASF em Petrolina $\mathrm{PE}$, que se localiza a latitude de $09^{\circ} 23^{\prime}$ sul, longitude de $40^{\circ} 30^{\prime}$ oeste e altitude de $376 \mathrm{~m}$. Segundo a classificação de Köppen, o clima da região apresenta-se como tropical semiárido, tipo BshW, caracterizado pela escassez e irregularidade das precipitações com chuvas no verão e forte evaporação em conseqüência das altas temperaturas.

O solo foi classificado como Argissolo Amarelo (EMBRAPA, 2006) que apresenta na camada de $0-20 \mathrm{~cm}$ de profundidade $85,9 \%$ de areia, $8,5 \%$ de silte e $5,6 \%$ de argila.

Como fonte de potência foi utilizado trator da marca: Valtra, modelo: 785 TDA (tração dianteira auxiliar), com $75 \mathrm{cv}(55,2 \mathrm{~kW})$, motor fabricado em 2004 com 3.100 horas de uso, com pneus dianteiros 12.4-24 R1 e traseiros 18.4 - 30 R1 em bom estado, e rotação de trabalho no motor a 2.040 RPM

Os equipamentos utilizados para condução do trabalho foram:

- Grade leve em Tandem segundo Stolf (2007), da marca, Marchesan TATU, modelo: GH, fabricada em 1999, com 7 discos em cada seção (quatro), sendo recortados na dianteira de 20" $(50,8 \mathrm{~cm})$ e lisos na traseira com mesmo diâmetro e distância entre discos de $19 \mathrm{~cm}$. Profundidade de trabalho de $0,10 \mathrm{~m}$.

- Grade leve Off-set (Figura 2b) segundo Stolf (2007), Marchesan TATU, modelo: GAM, fabricada em 1994, com 8 discos em cada seção (duas), sendo recortados de 22" $(55,8 \mathrm{~cm})$ e distância entre discos de $23 \mathrm{~cm}$. Profundidade de trabalho de $15 \mathrm{~cm}$, na abertura total e $0,10 \mathrm{~m}$ com $50 \%$ de abertura.

- Grade leve Off-set (Figura 2c) segundo Stolf (2007), Marchesan TATU, modelo: ATCR, fabricada em 2005, com 7 discos em cada seção (duas), sendo recortados de 24" $(60,9 \mathrm{~cm})$ e distância entre discos de $23 \mathrm{~cm}$. Profundidade de trabalho de $0,20 \mathrm{~m}$.

- Escarificador da marca Marchesan TATU, modelo: AST, fabricado em 2005 com 3 hastes e ponteira estreita de $5 \mathrm{~cm}$. Profundidade de trabalho de 0,30 m.

- Roçadora da marca Marchesan TATU, modelo: RC² 1300, fabricado em 2005 com duas facas móveis e sistema de embreagem para evitar esforços no eixo cardan. Largura de trabalho de 1,30 m.

- Adubadora da marca VICON, modelo PS 603, com dosador gravitacional e distribuidor pendular, acionamento pela tomada de potência.

Os níveis de potência sonora foram determinados por meio do decibelímetro digital 
da marca Minipa - modelo MSL-1325 com três faixas de medição (Baixa - 32-80 dB; 50-100 $\mathrm{dB}$ e 80-130 dB) e protetor de vento atendendo a norma IEC 651 (International Electrotechnical Commission), tipo 2 (classes de 0 a 3), que define para uso de campo. Foi utilizado conforme MTE (2008) nos circuitos de resposta lenta (SLOW) e de equalização "A", sendo expresso em dB.

As avaliações basearam-se no método descrito na NBR-9999 (ABNT, 1987). Segundo essa norma, na posição e momento do ensaio de medição do nível de potência sonora, a temperatura ambiente deve estar entre -5 e $30^{\circ} \mathrm{C}$ e a velocidade do vento devem ser inferior a $5,0 \mathrm{~m} \mathrm{~s}^{-1}$, que foram observados por meio de uma estação meteorológica no Centro de Ciências Agrárias. No dia do experimento (10/02/2010) a velocidade do vento e a temperatura eram de, $27,0^{\circ} \mathrm{C}$ e $5,3 \mathrm{~m} \mathrm{~s}^{-1}$, respectivamente, atendendo as solicitações da norma. Como a velocidade do vento estava próxima do limite, para o aparelho que atende a norma IEC 651 utilizou-se o protetor de vento.

Para realizar as medições, colocou-se o medidor de nível de potência sonora próximo ao ouvido do operador, entre $79 \mathrm{~cm}$ acima, $15 \mathrm{~cm}$ à frente e $20 \mathrm{~cm}$ lateralmente em relação ao ponto de referência do assento - (SIP - Seat Index Point), de acordo com a norma NBR 5353 (ABNT, 1999). Ou seja, o microfone foi instalado do lado da cabeça do operador que foi submetido ao nível de pressão sonora (MIALHE, 1996). Assim, as determinações foram coletadas a cada $5 \mathrm{~s}$.

Foram tomadas três medidas do nível de potência sonora no percurso de $30 \mathrm{~m}$ com o trator em movimento nas velocidades teóricas de $3,0 \mathrm{~km} \mathrm{~h}^{-1}$ (L1), 5,0 $\mathrm{km} \mathrm{h}^{-1}$ (L2), 8,7 $\mathrm{km} \mathrm{h}^{-1}$ (H1), 10,7 $\mathrm{km} \mathrm{h}^{-1}$ (L3) e 13,0 $\mathrm{km} \mathrm{h}^{-1}$ (H2), (adaptado de MIALHE, 1996) com o motor a $2.040 \mathrm{rpm}$ com o aparelho IEC 651.

Para a avaliação comparativa de todos os equipamentos em função das velocidades de deslocamento utilizou-se o delineamento inteiramente casualizado (DIC) em fatorial de dois fatores, sendo seis conjuntos mais a testemunha e cinco velocidades de deslocamento.

Os dados foram analisados por meio da estatística clássica, quando o teste de $\mathrm{F}$ foi significativo a pelo menos $5 \%$ de probabilidade aplicou-se o teste de Tukey para comparação das médias.

\section{RESULTADOS E DISCUSSÃO}

Na Tabela 1 é apresentado à síntese da análise de variância para o nível de potência sonora dos conjuntos e a velocidade de deslocamento. Verifica-se que os maiores valores de potência sonora foram observados para o trator com grade leve off-set com discos de 55,8 cm, 
e menor para o conjunto trator com roçadora. A velocidade não apresentou efeito significativo. No entanto, a interação conjunto $\mathrm{x}$ velocidades foi significativa, sendo apresentada na Tabela 2.

Tabela 1 - Síntese da análise de variância dos valores médios do nível de potência sonora (dB (A)) em função dos conjuntos, velocidade de deslocamento do conjunto trator-adubadora e o tempo de exposição.

\begin{tabular}{|c|c|c|}
\hline Fatores & $\begin{array}{l}\text { Nível de potência } \\
\text { sonora }(\mathbf{d B}(\mathbf{A}))\end{array}$ & $\begin{array}{l}\text { Tempo de Exposição } \\
\text { (H) }\end{array}$ \\
\hline \multicolumn{3}{|l|}{ CONJUNTOS (C) } \\
\hline Trator & $93,9 \mathrm{BC}$ & $2 \mathrm{~h} 15 \mathrm{~min}$ \\
\hline Trator+grade leve em tandem & $93,3 \mathrm{C}$ & $2 \mathrm{~h} 32 \mathrm{~min}$ \\
\hline Trator+grade leve off-set $55,8 \mathrm{~cm}$ & $95,9 \mathrm{~A}$ & 1h $45 \mathrm{~min}$ \\
\hline Trator+grade leve off-set $60,8 \mathrm{~cm}$ & $94,2 \mathrm{~B}$ & $2 \mathrm{~h} 12 \mathrm{~min}$ \\
\hline Trator+escarificador & $92,0 \mathrm{D}$ & $3 \mathrm{~h}$ \\
\hline Trator+roçadora & $91,1 \mathrm{E}$ & $3 \mathrm{~h} 27 \mathrm{~min}$ \\
\hline Trator+adubadora & $92,2 \mathrm{D}$ & $2 \mathrm{~h} 56 \mathrm{~min}$ \\
\hline \multicolumn{3}{|l|}{ VELOCIDADES (V) } \\
\hline 3,0 & 93,3 & $2 \mathrm{~h} 32 \mathrm{~min}$ \\
\hline 5,0 & 93,0 & $2 \mathrm{~h} 40 \mathrm{~min}$ \\
\hline 8,7 & 93,5 & $2 \mathrm{~h} 27 \mathrm{~min}$ \\
\hline 10,7 & 93,1 & $2 \mathrm{~h} 37 \mathrm{~min}$ \\
\hline 13 & 93,3 & $2 \mathrm{~h} 32 \mathrm{~min}$ \\
\hline \multicolumn{3}{|l|}{ TESTE DE F } \\
\hline $\mathrm{C}$ & $87,2 * *$ & -- \\
\hline $\mathrm{V}$ & $1,6 \mathrm{~ns}$ & -- \\
\hline $\mathrm{C} \times \mathrm{V}$ & $5,2 * *$ & -- \\
\hline $\mathrm{CV}$ & 0,7 & -- \\
\hline
\end{tabular}

Médias seguidas de mesma letra na coluna não diferem pelo Teste de Tukey. ${ }^{\text {ns}}$ : não significativo $(\mathrm{P}>0,05)$; *: significativo $(\mathrm{P} \leq 0,05)$; **: significativo $(\mathrm{P} \leq 0,01)$. CV.: coeficiente de variação $(\%)$.

Verifica-se para a velocidade de $3,0 \mathrm{~km} \mathrm{~h}^{-1}$ os maiores valores para o trator (testemunha) e o conjunto trator com grade leve off-set com discos de 55,8 cm e 60,8 cm. O uso de equipamentos de tração que exigem maior demanda de potência do motor pode estar associado a maior emissão de potência sonora. Zoppello et al. (1995) afirmam que nos implementos tracionados, o aumento no nível de potência sonora é devido à maior exigência de potência do motor e nas máquinas acionadas pela tomada de potência o aumento é causado pelos órgãos acionados. Fernandes (2003) de maneira semelhante afirma que quanto à operação agrícola executada foi com a roçadora apresentou os maiores níveis de potência sonora devido foram causado pelo implemento, enquanto que nas operações de preparo do solo, a subsolagem e a aração se mostraram mais ruidosas em razão dos grandes esforços do motor, requeridos pelo implemento. 
Tabela 2 - Síntese do desdobramento da interação para os valores médios do nível de potência sonora $(\mathrm{dB}(\mathrm{A}))$ em função dos conjuntos e velocidade de deslocamento.

\begin{tabular}{llllll}
\hline Conj. & \multicolumn{5}{c}{ Velocidade (km h-1 $)$} \\
\cline { 2 - 6 } & \multicolumn{1}{c}{$\mathbf{3 , 0}$} & \multicolumn{1}{c}{$\mathbf{5 , 0}$} & \multicolumn{1}{c}{$\mathbf{8 , 7}$} & \multicolumn{1}{c}{$\mathbf{1 0 , 7}$} & \multicolumn{1}{c}{$\mathbf{1 3 , 0}$} \\
\hline 1 & $94,7 \mathrm{Aa}$ & $94,5 \mathrm{ABab}$ & $93,0 \mathrm{Bbc}$ & $92,6 \mathrm{BCc}$ & $94,6 \mathrm{ABa}$ \\
2 & $92,3 \mathrm{Bb}$ & $93,1 \mathrm{BCab}$ & $93,0 \mathrm{Bab}$ & $94,2 \mathrm{Ba}$ & $93,7 \mathrm{BCab}$ \\
3 & $95,8 \mathrm{Aa}$ & $95,8 \mathrm{Aa}$ & $96,4 \mathrm{Aa}$ & $96,5 \mathrm{Aa}$ & $95,4 \mathrm{Aa}$ \\
4 & $95,3 \mathrm{Aab}$ & $93,7 \mathrm{Bbc}$ & $96,0 \mathrm{Aa}$ & $93,3 \mathrm{BCc}$ & $92,7 \mathrm{CDEc}$ \\
5 & $91,6 \mathrm{Bb}$ & $91,8 \mathrm{CDb}$ & $91,6 \mathrm{BCb}$ & $91,8 \mathrm{CDb}$ & $93,4 \mathrm{BCDa}$ \\
6 & $91,8 \mathrm{Ba}$ & $91,0 \mathrm{Da}$ & $91,2 \mathrm{Ca}$ & $90,5 \mathrm{Da}$ & $91,2 \mathrm{Ea}$ \\
7 & $91,7 \mathrm{Bab}$ & $91,6 \mathrm{CDb}$ & $93,2 \mathrm{Ba}$ & $92,6 \mathrm{BCab}$ & $91,9 \mathrm{DEab}$ \\
\hline
\end{tabular}

Legenda: 1.Trator; 2.Trator+grade leve em tandem; 3.Trator+grade leve off-set 55,8 cm; 4.Trator+grade leve off-set $60,8 \mathrm{~cm}$; 5.Trator+Escarificador; 6.Trator+roçadora; 7.Trator+adubadora. Médias seguidas de mesma letra minúscula na LINHA e maiúscula na COLUNA não diferem entre si pelo teste de TUKEY a $5 \%$ de probabilidade.

O conjunto trator grade leve com discos de $55,8 \mathrm{~cm}$ apresentou o maior nível de potência sonora em todas as velocidades, apenas nas velocidades de 5,0;8,7 e 13,0 $\mathrm{km} \mathrm{h}^{-1}$, foi similar ao trator, conjunto trator grade leve off-set com discos de $60,8 \mathrm{~cm}$, respectivamente.

O nível de potência sonora para a velocidade dentro de cada conjunto independentemente indicaram para a maioria dos conjuntos, maiores valores nas maiores velocidades. O maior nível de potência sonora para o trator foi encontrado nas marchas mais longas (VITÓRIA, 2000).

Santos Filho et al. (2003) realizaram um estudo com o objetivo de determinar os níveis de potência sonora emitidos por um trator agrícola executando a operação de gradagem em diferentes velocidades de trabalho, com o trator de potência de 55,2 kW (75 cv) acoplado a uma grade leve (niveladora) de 28 discos. Concluíram que o sistema trator implemento emitiu níveis de potência sonora acima do limite de $85 \mathrm{~dB}$ (A) para uma jornada de 8 horas diárias, variando entre 92,9 a 96,8 dB (A). Valores pouco acima dos encontrados neste trabalho.

Os menores valores do nível de potência sonora foram observados nos conjuntos trator com a roçadora e em algumas velocidades trator com o escarificador e trator com a adubadora pendular.

Segundo Zoppello et al. (1995), os valores que mais poderiam causar danos à saúde, são aqueles registrados com o trator tracionando o arado e a grade de discos, que aumentam os níveis de potência sonora devido a maior exigência de potência do motor, e força de tração. Verifica-se, de fato, a relação da diminuição do tempo de exposição do operador para o conjunto 3 (trator-grade $55,8 \mathrm{~cm}$ ) e aumento da média do nível de potência sonora em relação aos demais conjuntos estudados, com tempos de exposição inferiores a $2 \mathrm{~h}$. 
Uma das maneiras de se diminuir o nível de potência sonora é com o uso de protetores auriculares. Assim, apesar de alguns modelos de protetores auriculares permitirem níveis de redução de potência sonora superior a 20 dB (A), Pessina e Guerretti (2000), concluíram que, em média, os dispositivos de proteção auricular permitem atenuação do nível de potência sonora na ordem de $10 \mathrm{~dB}(\mathrm{~A})$.

Segundo Barbosa, Barbosa e Volpato (2009) o Instituto Nacional Americano de Padronização (ANSI S 12.6 de 1997) define os parâmetros de atenuação para três classificações de protetores auditivos: protetores do tipo concha, atenuação de $75 \%$, para protetores de inserção automoldáveis $50 \%$ e para todos os outros tipos de protetores, $30 \%$ de atenuação.

Na Tabela 3 é apresentado o tempo de exposição médio em função dos níveis de potência sonora para as velocidades e os conjuntos. Considerando os valores de potência sonora acima de $85 \mathrm{~dB}(\mathrm{~A})$ o tempo de exposição fica reduzido em todas as operações, o que demonstra a necessidade de proteção auricular em todas as operações e nas diferentes velocidades.

Tabela 3 - Tempo de exposição (TE - horas) para os valores médios do nível de potência sonora (dB (A)) em função dos conjuntos e da velocidade de deslocamento do conjunto.

\begin{tabular}{|c|c|c|c|c|c|c|c|c|c|c|}
\hline \multirow[t]{2}{*}{ Conj. } & \multicolumn{10}{|c|}{ Velocidade (km h-1) } \\
\hline & $3, \mathbf{0}$ & TE & $\mathbf{5 , 0}$ & TE & 8,7 & TE & 10,7 & TE & 13,0 & TE \\
\hline 1 & 94,7 & 2h 04min & 94,5 & 2h 07min & 93,0 & 2h 40min & 92,6 & $2 \mathrm{~h} 48 \min$ & 94,6 & $2 \mathrm{~h} 06 \mathrm{~min}$ \\
\hline 2 & 92,3 & $2 \mathrm{~h} 54 \mathrm{~min}$ & 93,1 & $2 \mathrm{~h} 37 \mathrm{~min}$ & 93,0 & $2 \mathrm{~h} 40 \mathrm{~min}$ & 94,2 & $2 \mathrm{~h} 12 \mathrm{~min}$ & 93,7 & $2 \mathrm{~h} 22 \mathrm{~min}$ \\
\hline 3 & 95,8 & 1h $52 \mathrm{~min}$ & 95,8 & 1h $52 \mathrm{~min}$ & 96,4 & 1h $36 \mathrm{~min}$ & 96,5 & 1h $42 \min$ & 95,4 & 1h $54 \mathrm{~min}$ \\
\hline 4 & 95,3 & 1h $55 \mathrm{~min}$ & 93,7 & $2 \mathrm{~h} 22 \mathrm{~min}$ & 96,0 & 1h $45 \mathrm{~min}$ & 93,3 & $2 \mathrm{~h} 31 \mathrm{~min}$ & 92,7 & $2 \mathrm{~h} 46 \mathrm{~min}$ \\
\hline 5 & 91,6 & $3 \mathrm{~h} 12 \mathrm{~min}$ & 91,8 & $3 \mathrm{~h} \mathrm{06min}$ & 91,6 & $3 \mathrm{~h} 12 \mathrm{~min}$ & 91,8 & $3 \mathrm{~h} 06 \mathrm{~min}$ & 93,4 & $2 \mathrm{~h} 30 \mathrm{~min}$ \\
\hline 6 & 91,8 & 3h $06 \mathrm{~min}$ & 91,0 & $3 \mathrm{~h} 30 \mathrm{~min}$ & 91,2 & $3 \mathrm{~h} 24 \mathrm{~min}$ & 90,5 & $3 \mathrm{~h} 45 \mathrm{~min}$ & 91,2 & $3 \mathrm{~h} 24 \mathrm{~min}$ \\
\hline 7 & 91,7 & $3 \mathrm{~h} 09 \mathrm{~min}$ & 91,6 & $3 \mathrm{~h} 12 \mathrm{~min}$ & 93,2 & $2 \mathrm{~h} 35 \mathrm{~min}$ & 92,6 & $2 \mathrm{~h} 48 \mathrm{~min}$ & 91,9 & $3 \mathrm{~h} 03 \mathrm{~min}$ \\
\hline
\end{tabular}

Legenda: 1.Trator; 2.Trator+grade leve em tandem; 3 .Trator+grade leve off-set de 55,8 cm; 4.Trator+grade leve off-set de 60,8 cm; 5.Trator+Escarificador; 6.Trator+roçadora; 7.Trator+adubadora.

Uma maneira de minimizar o efeito do nível de potência sonora e aumentar o tempo de exposição é por meio das cabines. Franklin et al. (2006) mostraram que as cabines nos tratores podem reduzir o nível de ruído em $16 \mathrm{~dB}(\mathrm{~A})$.

\section{CONCLUSÃO}

Pelos resultados obtidos e nas condições em que foram desenvolvidas este trabalho, conclui-se que:

Os níveis de potência sonora em todos os conjuntos estudados estavam acima de 85 dB, 
considerando uma jornada de trabalho de 8 h, estabelecido pela NR-15 e os operadores estão sujeitos a elevados níveis de potência sonora, sendo indispensável o uso de protetores auriculares, ou que todos os projetos de tratores sejam providos de cabine que pode atenuar consideravelmente o nível de potência sonora, contribuindo para diminuir a insalubridade da operação.

O trator (testemunha) e as operações de gradagem obtiveram os maiores níveis de potência sonora.

Os equipamentos acionados pela fonte de potência (roçadora e adubadora) apresentaram níveis de potência sonora menor do que o trator e as demais operações.

\section{REFERÊNCIAS}

ABNT- ASSOCIAÇÃO BRASILEIRA DE NORMAS TÉCNICAS. NBR 9999: medição do nível de potência sonora, no posto de operação de tratores e máquinas agrícolas. Rio de Janeiro, 1987. 21p.

BARBOSA, M.A.G.; BARBOSA, J.A.; VOLPATO, C.E.S. Antropometria em operadores de máquinas agrícolas e avaliação ergonômica das máquinas agrícolas em sistemas de produção diversificada. In: Congresso de Extensão da UFLA - CONEX, 4, 2009, Lavras. Anais.... Lavras: UFLA, 2009. P.1-18.

DELMOND, J.G.; REIS, E.F. Avaliação de níveis de ruído emitidos por tratores em diferentes operações agrícolas. In: Seminário de Iniciação Científica, 4, 2006, Anápolis. Anais... Anápolis: UEG, 2006. p.7-12. Cd-Rom.

EMBRAPA - EMPRESA BRASILEIRA DE PESQUISA AGROPECUÁRIA. Sistema Brasileiro de Classificação de Solos. Brasília: Centro Nacional de Pesquisas de Solos, 2006. 370p.

FERNANDES, J.C. Levantamento estatístico sobre o nível de ruído em operações agrícolas com tratores. In: Congresso Brasileiro de Engenharia Mecânica, 12, 1993, Brasília, Anais... Brasília: Cobem, 1993.

FERNANDES, J.C. Tratores - ruídos: barulho ensurdecedor. Cultivar Máquinas, Pelotas, v.17, p.6-8, 2003.

FRANKLIN, R. C. et al. Factors affecting farm noise during common agricultural activities. Journal of Agricultural Safety and Health, St. Joseph, v. 12, n. 2, p. 117-125, 2006.

MARTINELLI, M.M. Medição da pressão sonora em tratores agrícolas novos e suas adequações à N.R. 15 da C.L.T. In: Fórum Ambiental da Alta Paulista, 4, 2008, Tupã. Anais... Tupã: ANAP, 2008. Cd_rom.

MIALHE, L.G. Máquinas agrícolas: ensaios e certificações. Piracicaba: FEALQ, 1996. $722 p$. 
MTE - MINISTÉRIO DO TRABALHO E DO EMPREGO. Atividades e operações insalubres: NR-15. Disponível em:

<http://www.mtb.gov.br/legislacao/normas_regulamentadoras/nr_15.asp> Acesso em 9 nov. 2008.

PESSINA, D.; GUERRETTI, M. Effectiveness of hearing protection devices in the hazard reduction of noise from used tractors. Journal of Agricultural Engineering Research, Silsoe, v. 75, n. 1, p. 73-80, 2000.

SANTOS FILHO, P. F. et al. Avaliação dos níveis de vibração vertical no assento de um trator agrícola de pneus utilizando um sistema de aquisição automático de dados. Revista Árvore, Viçosa, v.27, n. 6, p. 887-895, 2003.

SIMONE, M. et al. El tractor agrícola: fundamentos para su selección y uso. In: CUNHA, J.P.A.; DUARTE, R.M.A.; RODRIGUES, V.J.C. Avaliação dos níveis de vibração e ruído emitidos por um trator agrícola em preparo de solo. Pesq. Agropec. Trop., Goiânia, v. 39, n. 4, p. 348-355, out./dez.2009.

STOLF, R. Grades agrícolas: 4- Nova classificação quanto à função no preparo do solo.

Alcoolbrás, São Paulo, n. 114, p.69-72. 2007.

VITÓRIA, E. L. Avaliação do nível de ruído emitido por tratores em diferentes operações agrícolas. 2000. 76p. Dissertação (Mestrado em Engenharia Agrícola) - Curso de Pós-graduação em Mecanização Agrícola, Universidade Federal de Viçosa.

ZOPPELLO, G.; MONARCA, D.; CECCHINI, M. Aziende agricole, il rischio da rumore ed il D.Lgs 277/91. Macchine e Motori Agricoli, Bologna, v.2, n.10, p. 9-16, 1995. 
